\title{
The 2017 annual conference of Geoinformatics was held at CPGIS's birthplace to celebrate 25 years' success
}

YE Xinyue ${ }^{1,2,3}$

1. Department of Geography, Kent State University, Kent, Ohio 44242, USA;

2. School of Digital Sciences, Kent State University, Kent, Ohio 44242, USA;

3. Department of Computer Science, Kent State University, Kent, Ohio 44242, USA

The 2017 CPGIS (The International Association of Chinese Professionals in Geographic Information Science) annual conference of Geoinformatics was held on August 2-4, 2017 in Buffalo, NY, USA. It was the 25th anniversary of CPGIS. The National Center for Geographic Information and Analysis at Buffalo (NCGIA-Buffalo, led by Director Dr. Ling Bian) and CPGIS organized the conference at CPGIS's birthplace to celebrate 25 years' success. Under the theme of "The Past and Future 25 Years", this event was also co-organized by the following units: CPGIS Base at College of Environment and Planning, Henan University; Confucius Institute, University at Buffalo; State Key Laboratory of Resources and Environmental Information System, Institute of Geographic Sciences and Natural Resources Research, Chinese Academy of Sciences; NSF Spatiotemporal Innovation Center, UCSB/GMU/Harvard; International Society for Digital Earth; Ministry of Education's Key Laboratory of Poyang Lake Wetland and Watershed Research, Jiangxi Normal University; Office of the Vice Provost for International Education, University at Buffalo; State Key Laboratory of Information Engineering in Surveying, Mapping and Remote Sensing, Wuhan University.

The Geoinformatics conference series was initiated by CPGIS in 1992. This international conference series has provided a unique forum for exchanging novel ideas and cutting-edge knowledge on geographic information sciences and technologies among GIS professionals worldwide. Established 25 years ago on the SUNY-Buffalo campus initiated by Hui Lin (CPGIS Founder and Inaugural President) and a group of students with big dreams, CPGIS has grown up to a mature association with members from 31 countries and regions. 19 former and current CPGIS presidents arrived at the Buffalo conference along with over 200 participants from all over the world: Hui Lin, Yuemin Ding, Bin Li, Lin Liu, Hanming Tu, Shuming Bao, Chuang Tao, Fahui Wang, A-Xing Zhu, Chaowei Yang, Le Wang, Yongmei Lu, Aijun Chen, Xun Shi, Shaowen Wang, Mei-Po Kwan, Shixiong Hu, Xinyue Ye, and Jianya Gong.

World-class academicians from National Academy of Sciences of Canada, China, UK, and USA delivered keynotes, including Luc Anselin (University of Chicago), Michael Batty (University College London), Jing. M. Chen (University of Toronto), Jianya Gong (Wuhan University), Michael Goodchild (University of California, Santa Barbara), Huadong Guo 
(Chinese Academy of Sciences), Deren Li (Wuhan University), and Anthony Yeh (University of Hong Kong), with the video speech from Guanhua Xu (Chinese Academy of Sciences).

This conference covered both traditional topics in GIScience and new and emergent themes including: Big GeoData, GIScience of the Internet of Things, Geospatial Semantic Web, Sensor Geomatics, Geosimulation Modeling, Mobile Computing, Mobile Behavior, Complex Networks, Online Social Media, Cyber Computing, Events and Process, and Critical GIS to address societal issues, such as climate change, smart cities, precision medicine, urban mobility, crime analysis, migration, disaster, and environmental sustainability. Topics on China were included such as population, urban development, and environment. Papers from a wide range of disciplines related to Geographic Information Sciences were also invited, including: Geography, Cognitive science, Computer science, Engineering, Information science, Social science, Urban Planning, among many others.

Many IEEE GRSS members have been actively involved in organizing and participated in the Geoinformatics conference series. In addition to this year, IEEE GRSS has been the technical sponsor for ten past Geoinformatics conferences, including Geoinformatics 2005 (Wuhan, China), Geoinformatics 2009 (Fairfax, USA), Geoinformatics 2010 (Beijing, China), Geoinformatics 2011 (Shanghai, China), Geoinformatics 2012 (Hong Kong, China), Geoinformatics 2013 (Kaifeng, China), Geoinformatics 2014 (Kaohsiung, Taiwan), Geoinformatics 2015 (Wuhan, China), and Geoinformatics 2016 (Galway, Ireland).

CPGIS has been committing to promoting the development of research, education and entrepreneurship in geographic information science and technology at all levels around the world. As a non-profit organization run by volunteers, CPGIS organizes an international conference of Geoinformatics every year in various countries and regions. It also has a quarterly and peer-reviewed academic journal, Annals of GIS, published by Taylor \& Francis.

The missions of CPGIS is as following:

(a) To promote the professional development of its members by fostering cooperation among its members and by maintaining a central point of reference and deliberation.

(b) To promote the exchange of ideas, knowledge, and scientific development in GIS and related sciences and technologies between Chinese GIS professionals abroad and those in China.

(c) To provide an efficient channel between its members and other GIS professionals, through which mutual understanding and cooperation can be enhanced.

(d) To promote education of geographic information sciences at all levels around the world.

CPGIS has established a unique platform for students, scholars and professionals in GIS to develop their social network, leadership and careers. With a fantastic journey over the past 25 years of CPGIS, CPGIS hopes to work towards a more remarkable achievement in the following years!

(This author is the President of CPGIS (2016-17), who is the Director of Computational Social Science Lab, Kent State University.) 